\title{
Fast Far-Field Estimation Method by Compact Single Cut Near-Field Measurements for Electrically Long Antenna Array
}

\author{
Yoshiki Sugimoto, Student Member, IEEE, Hiroyuki Arai, Fellow, IEEE, Toshiyuki Maruyama, Masahiko Nasuno, \\ Masanobu Hirose, and Satoru Kurokawa
}

\begin{abstract}
This article proposes a fast far-field estimation method of electrically long antenna in compact measurement space. The presented method estimates a far-field pattern on an orthogonal cut plane from the linear equivalent electric current and circular current distribution. The equivalent electric current data is measured in a reactive near-field region using a small loop probe. In the proposed method, the linear equivalent current distribution is extended by a simple numerical extrapolation formula for improving the accuracy of the estimated far-field in the broadside direction. In the horizontal plane, the far-field pattern is calculated from both the circular equivalent electric and magnetic distribution approximating equivalent magnetic current with equivalent electric current. We confirmed the validity of the proposed method from the numerical simulation and measurements results. The method allows accurate directivity measurement in a small space in about a few minutes.
\end{abstract}

Index Terms-Near-field measurement, Far-field estimation, Equivalent electric current, Electrically long antenna, Reactive near-field.

\section{INTRODUCTION}

$\mathbf{S}$ IMPLE and accurate measurement method for base station antenna is necessary because the number of base stations installed is increasing because of the rapid development of mobile communications. Far-field is one of the most important characteristics to evaluate the performance of a base station antenna. Long distance between the antenna and the probe is required for measurement because it needs to satisfy the farfield criteria of electrically large aperture antennas [1], [2]. It is difficult to measure the far-field and its directivity of a base station antenna in a widely used anechoic chamber to measure a handset antenna, because it has an electrically long aperture constructed using multiple elements [3], [4].

The near-field measurement technique is an effective method to characterize far-field of an antenna under test (AUT) [5]. The cylindrical near-field measurement is the most commonly adopted technique for a base station antenna as it is suitable to transform a fan beam pattern. Reducing the time of

Y. Sugimoto is with the Department of Electrical and Computer Engineering, Graduate School of Engineering, Yokohama National University, Yokohama, Japan. e-mail: yosiki0505@gmail.com.

H. Arai is with the Department of Electrical and Computer Engineering, Yokohama National University, Yokohama, Japan email: arai@ynu.ac.jp.

T. Maruyama and M. Nasuno are with the NAZCA Ltd., Suwa, Japan.

M. Hirose and S. Kurokawa are with the Advanced Industrial Science and Technology, Tsukuba, Japan.

Manuscript received May 18, 2017. measurement and calculation, an antenna is crucial for nearfield measurements [6]. There are some presented methods that resolve this problem. Multi-probe measurement is one of the simple techniques for resolving this problem [7]. Measurement time is reduced drastically by introducing a large number of probes to the equipment. Another is the single cut nearfield to far-field transformation. Processing time achieved is minimized by limiting the measurement data and the estimate plane. In literature [6], [8], methods to reconstruct far-field accurately by circular one cut measurements and cylindrical modes transformation are shown. However, in most cases, it is difficult to rotate the AUT to measure the broadside directivity of the long aperture AUT. The cylindrical setup requires smaller measurement space in long antenna measurement than for the spherical setup, as the AUT is rotated only in the azimuthal direction. The cylindrical one-cut near-field measurement is most suitable for obtaining quickly the farfield of a base station antenna in a compact anechoic chamber.

The cylindrical scanning system has a defect because of its mechanical structure, which is a finite truncation of the longitudinal direction. In the cylindrical systems, it is impossible to acquire an infinitely lengthy data in the endfire direction; thus, an estimable angle of far-field pattern is limited. To avoid this problem, only numerical extrapolation as in [9] is possible. Valid angle of the far-field is increased in [9], however, the measurement of the length of the cylinder is desirable, which can be reduced to at least the same length as the AUT. If the measured length is longer than the AUT length, the apparatus becomes overly complicated. In particular, in the case of the single cut measurement, as complicated numerical processing can not be performed from a small amount of acquired data, there is a need to extrapolate the assumed value to be as simple as possible.

In this article, we propose a rapid far-field estimation method by single cut and short length near-field measurement for an electrically long array antenna. The proposed method to estimate the far-field is based on equivalent line electric current, which is measured in reactive near-field using a small loop probe to minimize disturbance of the electromagnetic field in the vicinity of the AUT. The equivalent currents are measured on a line in front of and in a circle around the AUT. The far-field pattern of each plane is estimated from the extended linear distribution and the circular distribution by apply the 1-D integral equation, the measurement time and the equipment can be kept minimal. The spherical far-field 
is approximated from the product of the far-field of each cut plane, and its maximum directivity can be estimated. A priority of the proposed method is fast measurement and a small scale of measurement space.

The article is organized as follows. Section II describes the estimation theory and the simple extrapolation method. Numerical test and its accuracy evaluation is provided in section III. Section IV validates the proposed method via the measured near-field data. Finally, conclusions are drawn in section $\mathrm{V}$.

\section{MethoD}

The proposed method follows the near-field to far-field transformation method based on the equivalent current method [10], [11]. The equivalent current theorem assumes an arbitrary surface enclosing the AUT. This approach can obtain the radiation field outside the closed surface if the equivalent current on the surface is determined by measurement. An equivalent electric current $\boldsymbol{J}$ and an equivalent magnetic current $M$ on the closed surface are obtained from the electromagnetic field on the closed surface as

$$
\begin{aligned}
& \boldsymbol{J}=\hat{\mathbf{n}} \times \boldsymbol{H} \\
& \boldsymbol{M}=\boldsymbol{E} \times \hat{\mathbf{n}}
\end{aligned}
$$

where $\hat{\mathrm{n}}, \boldsymbol{H}$, and $\boldsymbol{E}$ are outward normal vector of AUT, magnetic field and electric field. To obtain the radiation field on an arbitrary point, $\boldsymbol{J}$ and $\boldsymbol{M}$ are necessary. However, to measure both the electric and magnetic fields, the measurement equipment is complex. The radiation field outside the closed surface is determined from either the electric current distribution or magnetic current if we can consider the internal radiation field of the closed surface to be zero. The closed surface is replaced by a perfect magnetic conductor (PMC) or a perfect electric conductor (PEC) and by introducing the Schelkunoff's theorem, the radiation field can be obtained from either $\boldsymbol{J}$ or $\boldsymbol{M}$ [11]-[15]. The proposed method estimates far-field using only equivalent electric current distribution. To obtain the equivalent current, a small loop probe with little electromagnetic field disturbance is used. As the electrical size of the probe is small enough, the disturbance can be kept less; hence, we measure the equivalent current close to the AUT. For simplifying the estimation technique, we assume the equivalent electric currents as an infinitesimal dipole group to calculate the far-field pattern.

The superiority of the proposed method for the ordinary cylindrical near-field to far-field transformation is the speeding up of measurement by reducing the number of measurement points. To reduce the number of measurement points, the proposed technique restricts the estimated plane only in the $\phi=0^{\circ}$ and $\theta=90^{\circ}$ cut plane. The geometry of the near-field measurement is shown in Fig. 1. The longitudinal direction of the AUT is set parallel in the $z$-axis direction. The vertical plane far-field pattern is estimated from the linear current distribution parallel to the longitudinal direction of the AUT [16]. We need to calculate only in the electromagnetic field in the region where $x>0$, we place a perfect magnetic conductor in front of the AUT extending to infinity in the $y$ and $z$ directions on the $y z$ plane. Therefore, the actual equivalent current distribution on the closed surface is given as $\boldsymbol{J}^{\prime}=$ $2 \hat{n} \times \boldsymbol{H}$ according to image theory [11]. In theory, the PMC must be assumed to be of infinite length; however, it cannot be realized physically. By focusing on the AUT, does not radiate to $\pm z$ direction, we assume a finite length linear PMC to be of the same length as the AUT and to obtain equivalent electrical current distribution over the line. The proposed method regards the equivalent current on each measurement point as a small electric dipole, and the radiation field from the dipole is calculated [17]. The far-field is calculated by integrating the radiation field from each dipole. Although each dipole is considered as an infinitesimal dipole of zero length, the far-field is calculated similar a linear array antenna [18] as follows:

$$
\begin{aligned}
& \boldsymbol{E}_{\theta}(\theta)=Z_{0} \sin (\theta) \sum_{m=1}^{M} \boldsymbol{J}_{z}^{\prime}(m) e^{\left(j k_{0} z_{m} \cos \theta\right)}, \\
& \boldsymbol{E}_{\phi}(\theta)=Z_{0} \sum_{m=1}^{M} \boldsymbol{J}_{y}^{\prime}(m) e^{\left(j k_{0} z_{m} \cos \theta\right)}
\end{aligned}
$$

where $Z_{0}$ is the characteristic impedance in free space, subscription means a polarization, $\boldsymbol{J}^{\prime}{ }_{z}(m)$ and $\boldsymbol{J}_{y}{ }_{y}(m)$ is the acquired complex current, $z_{m}$ is its position on the $z$-direction, and $k_{0}$ is the wavenumber in free space.

To measure in as compact an area as possible, the measurement length of the linear current distribution is assumed to be equal to the length of the AUT as a precondition. Valid angle in broadside region is zero according to the definition [9], and the far-field pattern in the vertical plane cannot be estimated accurately. Truncation error reduction method was denoted as in [9], although it requires a longer measurement than the length of the AUT, and a complex reconstruction algorithm. As the method is insufficient to reduce the estimation time, it is necessary to apply a simpler extrapolation method. The sectional view of the linear current sampling and extrapolation scheme is shown in Fig. 2. As previously mentioned, the actual measurement area has the same length as the AUT $l_{M}$, the upper and the lower data is extended using the data of the measured edge point $J\left(l_{\mathrm{M}} / 2\right)$, where $\left(l_{\mathrm{M}} / 2\right)$ means coordinates position on the $z$-axis. The extended currents approximated from the amplitude are inversely proportional to the distance and the phase rotation corresponding to distance is given as

$$
\begin{aligned}
J\left(\frac{L_{\mathrm{M}}}{2}+\Delta z_{i}\right) & =J\left(\frac{L_{\mathrm{M}}}{2}\right) A_{\Delta z i} e^{\left(-j k_{0} d_{\Delta z i}\right)} . \\
A_{\Delta z i} & =\frac{d^{2}+\left(\frac{L_{\mathrm{M}}}{2}\right)^{2}}{d^{2}+\left(\frac{L_{\mathrm{M}}}{2}+\Delta z_{i}\right)^{2}}, \\
d_{\Delta z i} & =\sqrt{d^{2}+\Delta z_{i}^{2}}-d .
\end{aligned}
$$

$\Delta z_{i}$ is distance between the edge of measurement area and the position of the extrapolation point in the $z$-axis direction. This correction factor utilizes the nature that the amplitude sharply decreases and the phase constant rotates uniformly out of the aperture area in the very near-field region. In case the extrapolated length is too long beyond necessity, and the 


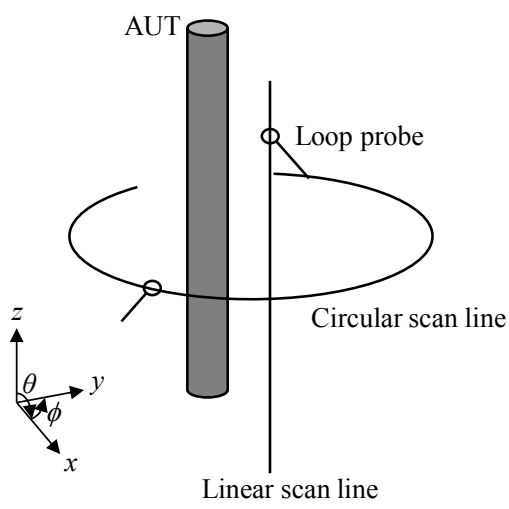

Fig. 1. Measurement setup of equivalent electric current in each single cut plane.

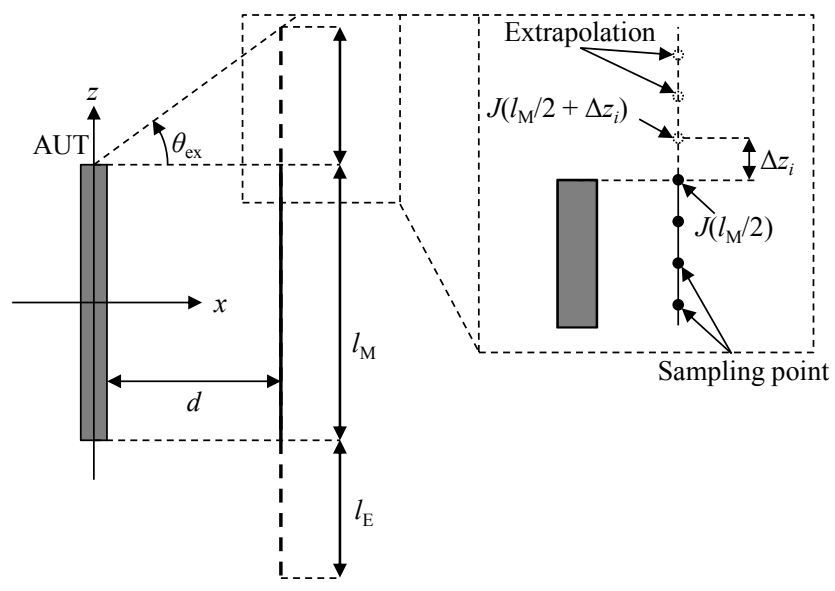

Fig. 2. Geometry of source extrapolation in vertical plane.

proportion of reliable data decreases, we need to limit the approximate length. Whatever the case, because the amount of measured data should not be less than the amount of extrapolated data, the extrapolated length $l_{\mathrm{E}}$ is limited to less than twice of measurement length $l_{\mathrm{M}}$ as $2 l_{\mathrm{E}} \leq l_{\mathrm{M}}$. It is confirmed that the far-field can be estimated accurately with this extrapolation length in many numerical calculations. By extending the linear equivalent current distribution twice as much as the actual measurement length, it is possible to extend the valid angle to

$$
\theta_{\mathrm{ex}}=\tan ^{-1}\left(\frac{l_{\mathrm{E}}}{d}\right) .
$$

It can reduce the error in the vertical plane because of the finite truncation of the linear current distribution. When extrapolation is not performed, the valid angle $\theta_{\mathrm{ex}}$ is $0^{\circ}$ and it is difficult to accurately obtain the pattern in the vertical plane.

The far-field in horizontal plane is calculated from the circular current distribution around the AUT. When we assume the closed surface to include the AUT, there is no need to consider the electromagnetic field in the closed surface in the vertical plane (only the external field of the closed surface is subject to calculation). In the vertical plane, the far-field pattern could be estimated accurately if either the equivalent electric current or equivalent magnetic current is known. However, with respect to the horizontal plane, we must consider an electromagnetic field in the direction that is inside the closed surface (e.g., an electromagnetic field in $\phi=180^{\circ}$ direction seen from the equivalent source on $\phi=0^{\circ}$ ). Both the equivalent electric and magnetic current distribution is necessary to accurately estimate the far-field. From the Maxwell's equations, the electric field is

$$
\begin{aligned}
\boldsymbol{E}= & j \omega \mu \boldsymbol{A}_{j}+\frac{1}{j \omega \epsilon} \nabla \nabla \cdot \boldsymbol{A}_{j} \\
& -\nabla \times \boldsymbol{A}_{m}
\end{aligned}
$$

where $\boldsymbol{A}_{j}$ and $\boldsymbol{A}_{m}$ are the electric and the magnetic vector potential defined as

$$
\begin{aligned}
\boldsymbol{A}_{j} & =\frac{1}{4 \pi} \int_{S} \boldsymbol{J}\left(\mathbf{r}^{\prime}\right) G\left(\mathbf{r}, \mathbf{r}^{\prime}\right) d S \\
\boldsymbol{A}_{m} & =\frac{1}{4 \pi} \int_{S} \boldsymbol{M}\left(\mathbf{r}^{\prime}\right) G\left(\mathbf{r}, \mathbf{r}^{\prime}\right) d S
\end{aligned}
$$

and

$$
G\left(\mathbf{r}, \mathbf{r}^{\prime}\right)=\frac{e^{-j k_{0}\left|\mathbf{r}-\mathbf{r}^{\prime}\right|}}{4 \pi\left|\mathbf{r}-\mathbf{r}^{\prime}\right|} .
$$

For the simplification of measurement equipment, it is hoped to be able to estimate far-field only from the equivalent electric current or the equivalent magnetic current. The proposed method uses only electric current. If calculated as above, the electromagnetic field inside the closed surface by equivalent current does not become zero. In other words, a perfect magnetic conductor cannot be assumed on the closed surface. Therefore, in (7), we approximate $\boldsymbol{M}=Z_{0} \hat{\mathrm{n}} \times \boldsymbol{J}$ [19]. Originally $\boldsymbol{M}=Z_{0} \hat{\mathrm{n}} \times \boldsymbol{J}$ does not hold in the very nearfield region. As the AUT has a width in the $y$-axis direction, the distance between the AUT and the probe is sufficiently separated around the main radiation direction when performing a circular scan. In this article, we assume that the distance from the AUT center to the circular scan line is farther than the wavelength. This is sufficient compared with the far-field criterion of an infinitesimal dipole. Thus the assumption holds well practically. Based on these, the horizontal plane far-field is calculated as follows;

$$
\begin{gathered}
\boldsymbol{E}_{\theta}(\phi)=Z_{0} \sum_{n=1}^{N}\left(1+\hat{\boldsymbol{\phi}} \cdot \hat{\boldsymbol{\phi}}_{\boldsymbol{n}}\right) \boldsymbol{J}_{z}(n) e^{\left(j k_{0} d_{\phi}\right)}, \\
\boldsymbol{E}_{\phi}(\phi)=Z_{0} \sum_{n=1}^{N}\left(1+\hat{\boldsymbol{\phi}} \cdot \hat{\boldsymbol{\phi}}_{\boldsymbol{n}}\right) \boldsymbol{J}_{\phi}(n) e^{\left(j k_{0} d_{\phi}\right)} . \\
d_{\phi}=x_{n} \cos \phi+y_{n} \sin \phi
\end{gathered}
$$

where $N$ is the total number of equivalent currents on the circle, $x_{n}$ and $y_{n}$ are the coordinate points on the $x$ - and $y$-axis, respectively, and, $\phi_{n}$ is the azimuth angle of each equivalent current. $\hat{\phi}$ is unit vector of $\phi$ direction and $\hat{\phi}_{n}$ is the position vector of each sampling point. The derivation is described in the appendix. 
By using the orthogonal cut planes obtained as described above, the spherical far-field pattern is approximated as

$$
\boldsymbol{E}_{p}(\theta, \phi)=\boldsymbol{E}_{p}\left(\theta, \phi=0^{\circ}\right) \boldsymbol{E}_{p}\left(\theta=0^{\circ}, \phi\right),(p=\theta, \phi),
$$

where $\boldsymbol{E}_{p}(\theta, \phi)$ is the virtually calculated full-sphere far-field, $\boldsymbol{E}_{p}\left(\theta, \phi=0^{\circ}\right)$ is the far-field in the vertical plane according to (2), and $\boldsymbol{E}_{p}\left(\theta=90^{\circ}, \phi\right)$ is far-field in horizontal plane by (9). The horizontal far-field pattern of electrically long antenna does not change significantly depending on the change of the zenith angle. Horizontal cut surface pattern for arbitrary $\theta$ can be approximated using single horizontal cut plane pattern of $\theta=90^{\circ}$. In addition, it is possible to estimate the vertical cut plane pattern with very high accuracy using the linear current distribution in front of AUT, because the radiation energy of the electrically long array antenna is mostly concentrated in the front region of the AUT. In other words, if the vertical and the horizontal cut plane patterns can be acquired, it is possible to characterize the spherical far-field pattern with sufficient accuracy. In the antenna evaluation for the base station, evaluation is performed mainly in each cut plane, and there is not much to be discussed for the full spherical pattern [20]. Using the above, the approximation accuracy of (9) holds well with respect to the long aperture antenna. Error in the vertical plane is derived from estimated normalized vertical plane pattern as

$$
\operatorname{Error}(\theta)=\frac{\left|F F_{\text {Ref. }}(\theta)-F F_{E s t .}(\theta)\right|}{\left|F F_{\text {Ref. }}\left(\theta_{0}\right)\right|},
$$

(12) is the function of the zenith angle $\theta$ and it can be obtained from the vertical plane pattern only. Where $F F_{\text {Ref. }}(\theta)$ is the exact far-field pattern, $F F_{E s t} .(\theta)$ is estimated far-field pattern, and $F F_{R e f .}\left(\theta_{0}\right)$ is the maximum value of the exact far-field in the vertical plane. We discuss the improved amount in the vertical plane using mean value of (12) in a valid angle area. We evaluate the total accuracy by the maximum directivity. The directivity in the maximum radiation direction $\left(\theta_{0}, \phi_{0}\right)$ is given by

$$
D\left(\theta_{0}, \phi_{0}\right)=\frac{4 \pi\left|E\left(\theta_{0}, \phi_{0}\right)\right|^{2}}{\int_{0}^{2 \pi} \int_{0}^{\pi}|E(\theta, \phi)|^{2} \sin \theta d \theta d \phi} .
$$

The method exerts its potency for estimate the directional gain pattern of sector beam antenna such as base station antennas with an electrically long aperture. This is because the method obtains the directional gain by approximating the full-spherical directional gain pattern from the product of orthogonal cuts pattern. Therefore, we assume that the shape of the AUT is electrically long and very thin as an important prerequisite. Under this condition, the present method can accurately estimate the far-field pattern in each cut plane and maximum directivity within the precision guaranteed by the measuring system. The developed method guarantees the estimation accuracy of each cut plane within the measurement accuracy combining both the antenna and gain standard [21] take account of the gain uncertainty of the reference antenna [22]. To evaluate absolute gain considering the loss of feeding circuit and others, it is necessary to apply antenna calibration techniques [23].

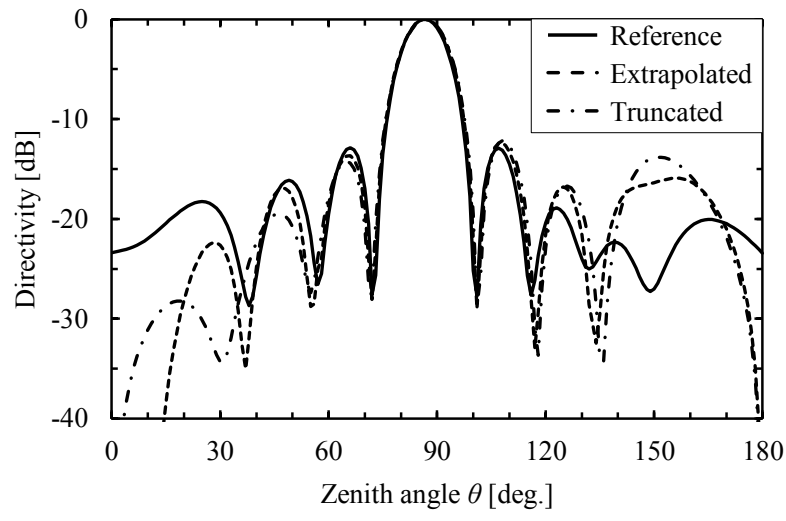

Fig. 3. Estimated vertical plane far-field sampled in very near-field, probe distance $d=0.2 \lambda$ (solid: reference; chain: truncated; dotted: extrapolated).

\section{NUMERICAL RESUlTS}

In this section, we demonstrate some numerical results by simulation of a long aperture linear array antenna. We used a four-elements patch linear array as an AUT (at a frequency of $3 \mathrm{GHz}$ ), its excitation is uniform, and it is arranged parallel to the $z$-axis direction. The AUT has a rectangular aperture of height $2 \lambda$ and width $\lambda$. Line current distribution is sampled at an interval of $0.1 \lambda$ right above the front of AUT and the circular current distribution is sampled at an interval of $5^{\circ}$ at the center of AUT. The actual sampling length of the linear current distribution is the same as the length of the AUT, and total length is extended twice as much as the length of the AUT according to (3). Although the sampling points are set densely here, it suffices if the interval meets the sampling theorem [5]. Therefore, the sampling interval can be shortened to be slightly shorter than a half wavelength.

The estimated far-field in the vertical plane from a nearfield data in very close region of the AUT is shown in Fig. 3. The horizontal axis of the figure denotes the zenith angle and the vertical axis denotes directivity. The distance between the AUT and the sampling line is $0.2 \lambda$. The co-polarization of the AUT is in the $\theta$ direction and the sampled current direction is parallel to $z$-axis. The solid line is the reference far-field pattern. The reference pattern is the exact far-field pattern of the AUT by full-wave simulation using FEKO [24]. In the case of Fig. 3, the estimated pattern of the truncated and the extrapolated show almost no difference. When the measurement distance is very close, a lack of the data in the endfire direction is not a big problem. However in many cases, because of the mechanical reason, it is physically impossible to deploy a probe in a very close range to the AUT. In particular, when evaluating characteristics with radomes, it can be inferred that the distance between the probe and AUT is about half a wavelength apart. The amount of data in the endfire direction is decreased as the distance of the linear current distribution is increased. As a result the estimation accuracy in the vertical plane far-field is degraded.

The extrapolated current distribution is shown in Fig. 4. The reference distribution is obtained by full-wave simulation. The vertical axes mean amplitude and phase of $z$ component 
equivalent current. Although the extrapolation well reproduces the exact current distribution for both amplitude and phase, it can be seen that when $|z|$ is sufficiently large, the extrapolated value is approximated larger than the exact amplitude. The true current decreases according to the sum of $1 / r$ and $1 / r^{2}$ terms depending on the distance from the AUT. However, because (3) decreases only with $1 / r$ with the measurement end as a starting point, an error from the exact value occurs at a portion where $|z|$ is sufficiently large. If the extrapolated length is infinite, the radiation level around the polar direction becomes hypertrophied. Therefore, we introduced $2 l_{\mathrm{E}} \leq l_{\mathrm{M}}$ as a limitation. As we can see from the pattern in the vertical plane, the method can estimate even an electrical tilt pattern.

The far-field pattern estimated from extrapolated near-field is shown in Fig. 5, when the measurement position $d=\lambda$. The vertical axis of Fig. 5 (b) denotes error function as in (12). As can be seen from Fig. 5 (a), the far-field obtained from the truncated near-field has a mismatch with the reference pattern in not only the side lobes but also in the main beam. In the case of a realistic measurement distance, missing data in the endfire direction cannot be avoided; the mismatch of the estimated pattern occurs. This pattern discrepancy is improved by the data extension, that is the valid angle of this sampling case is expanded to $\pm 60^{\circ}$ from $\pm 0^{\circ}$ without data extrapolation according to (5). It can be seen that the estimated pattern based on the extended data has good agreement with the reference far-field. This is apparent from Fig. 5 (b) in which the mean value of the error in the valid angle region is improved by more than $6 \mathrm{~dB}$. Fig. 6 shows the estimation results of the farfield in the case of the AUT radiating horizontal polarization. The AUT is a four-elements linear array, which is the same as the vertical polarization case. It is constructed by patch elements, which is radiating horizontal polarization. Even in horizontal polarization, the far-field pattern in the vertical plane can be accurately estimated by the proposed method, and particularly good agreement is obtained in the estimation result by extrapolating the near-field data. It was numerically demonstrated that proposed method can accurately estimate the far-field in the vertical plane. In a standard array antenna with small beam tilt, the maximum directivity in the vertical plane can be estimated accurately enough if the equivalent current is extrapolated to about $l_{\mathrm{E}} / l_{\mathrm{M}}=0.25$. Considering the case where a slightly large tilt is applied, it is recommended that the extrapolation length be $l_{\mathrm{E}} / l_{\mathrm{M}}=0.5$ with a margin. As the acquired distance of the linear current distribution is closer to the AUT, the extrapolation length can be shortened.

The horizontal plane pattern of the AUT estimated according to (9) is shown in Fig. 7. The figures present the copolarization pattern. Fig. 7 (a) is the pattern of the vertical polarized AUT such as in Figs. 3 and 5, and Fig. 7 (b) is the horizontal polarized AUT pattern such as in Fig. 6. The difference in line type indicates the difference in sampling radius $r$ from the AUT center. The estimation accuracy in the horizontal plane is wrong, when the sampling position of the scanning circle is too close to the AUT. The accuracy deterioration of the proposed method occurs from imperfection of the integral equation in near-field region. If the scan radius is sufficiently large, almost the same pattern as the

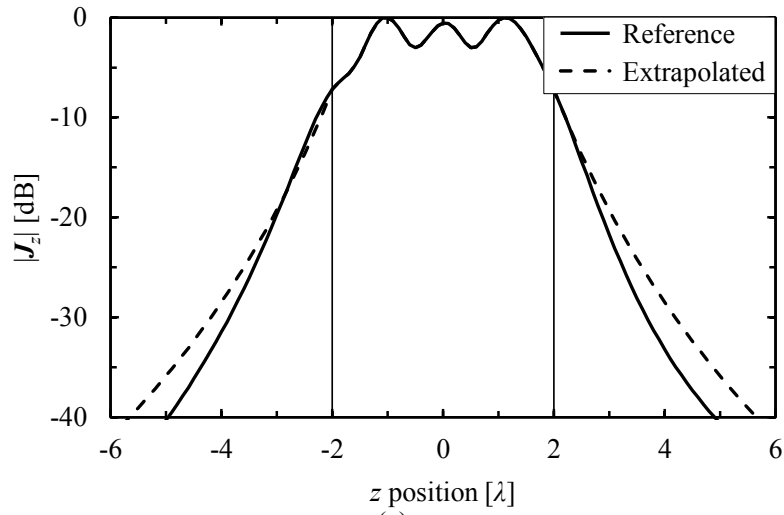

(a)

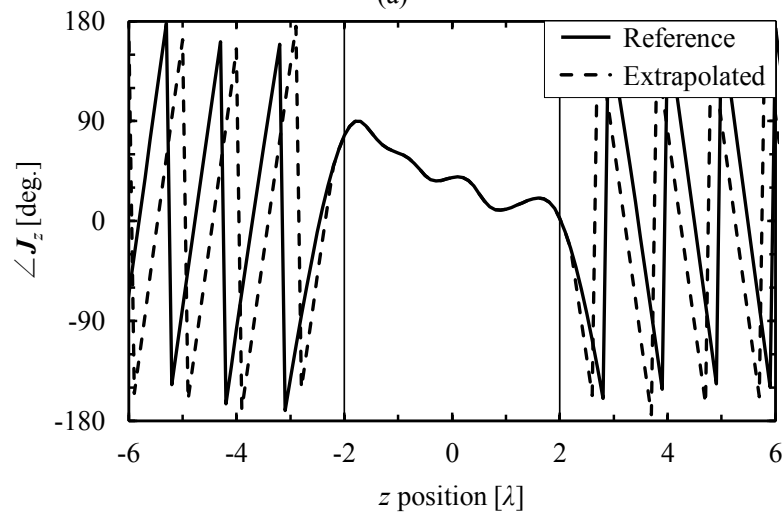

(b)

Fig. 4. Extrapolated equivalent current. (a) is amplitude (solid: reference; dotted: extrapolated). (b) is the phase (solid: reference; dotted: extrapolated). The vertical lines are its edge position of the AUT.

far-field is obtained, thus improving the estimation accuracy. To summarize measurement conditions, estimation accuracy in the vertical cut plane is better as the measured distance of the linear current distribution is closer to the AUT. On the other hand, large measurement radius of the circular current distribution is better for accurately estimating the far-field in the horizontal cut plane. To accurately estimate the pattern in the horizontal plane, it is desirable to provide a distance larger than about $0.5 \lambda$ between the edge in the horizontal plane of the AUT and the scan circle [25].

\section{EXPERIMENT}

This section shows estimation results using measured equivalent current in the very near-field area. A measurement overview of a base station antenna in an anechoic chamber is shown in Fig. 8. The measurement of the current distribution was performed in a small anechoic chamber. As shown in the previous section, as the proposed method can estimate the farfield using the equivalent current in the very near-field region of AUT, a large-scale anechoic chamber is unnecessary for near-field measurement. The outer diameter of the probe is 12 $\mathrm{mm}$, and the electrical size of the probe is about $0.1 \lambda$ at 2.69 $\mathrm{GHz}$. We have estimated the far-field without probe correction, as we are using a probe that is electrically small enough and it has an omni-directional pattern. The probe slides in the $z$ 


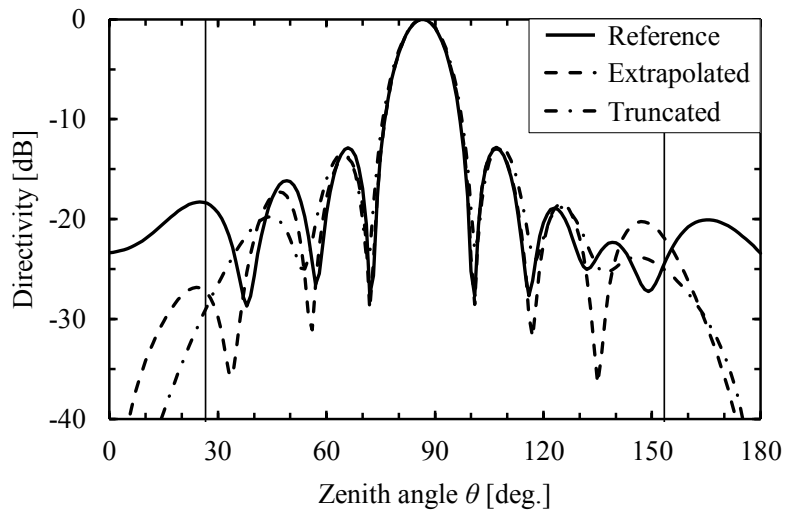

(a)

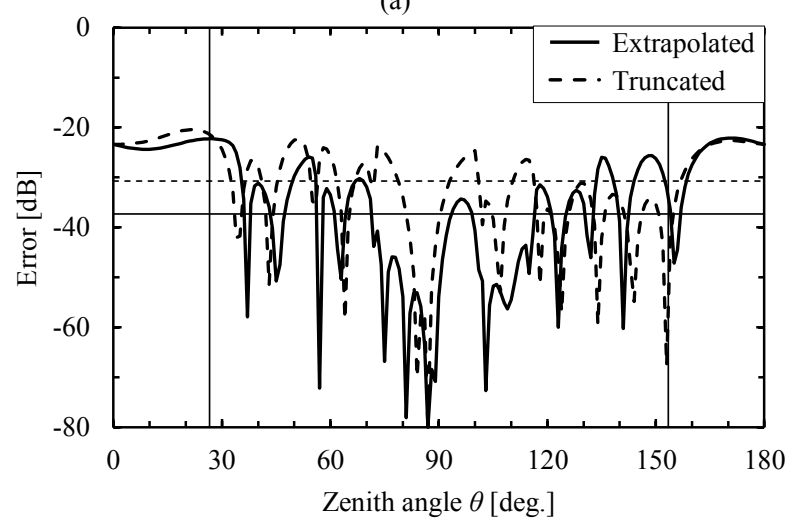

(b)

Fig. 5. Estimated results of $E_{\theta}$ in vertical plane, probe distance $d=\lambda$. (a) is the far-field pattern (solid: reference; chain: truncated; dotted: extrapolated). (b) is the relative error (solid: extrapolated; dotted: truncated). The horizontal lines in (b) present mean value in valid angle region and the region between vertical lines is valid angle region.

direction and the AUT rotates in the $x y$ plane. The AUT is configured by eight radiation elements placed on a co-linear line and the polarization is slanted at $45^{\circ}$. The scanning probe is tilted at $45^{\circ}$ from the vertical axis of the AUT. Measurement specifications of the current distribution are shown in Table I, where measurement length is $2155 \mathrm{~mm}$ for $900 \mathrm{MHz}$ band AUT and $834 \mathrm{~mm}$ for $2.6 \mathrm{GHz}$ band AUT; these are the same length as the physical length of each AUT. Measurement distance of the linear current distribution is set to $100 \mathrm{~mm}$, which is an approach limit of the probe jig. All measurements of the current distribution were performed in the very nearfield area of the AUT. The probe scans in a straight line above the AUT, and the AUT is held by the jig that can rotate in the circumferential direction. Compared to the cylindrical scan, the measurement time of the proposed method is extremely small, and the time required for the measurement was about 3 minutes.

Estimation results from the measured data are shown in Figs. 9 and 10. Measurement frequency is at $0.79 \mathrm{GHz}$ and 2.69GHz. The solid lines present the reference far-field pattern by near-field to far-field transformation using cylindrical measurement [26], and the vertical lines in the vertical plane present the range of the valid angle. The estimated pattern can reproduce the main lobe, especially when using the

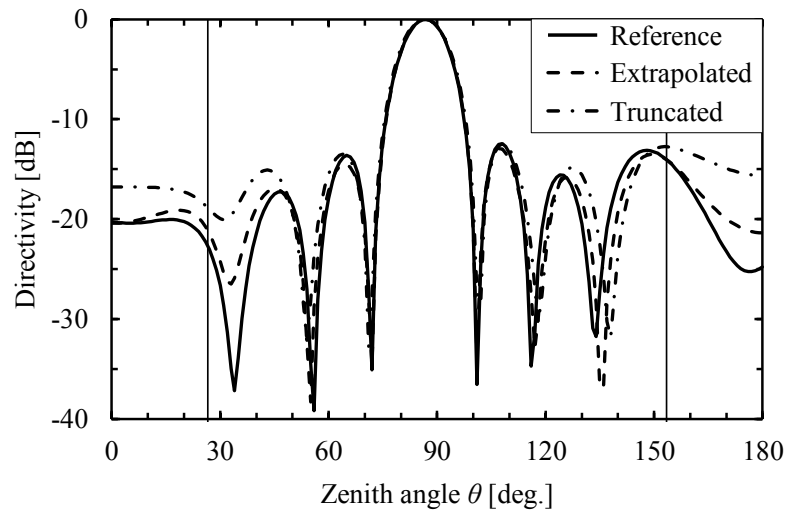

(a)

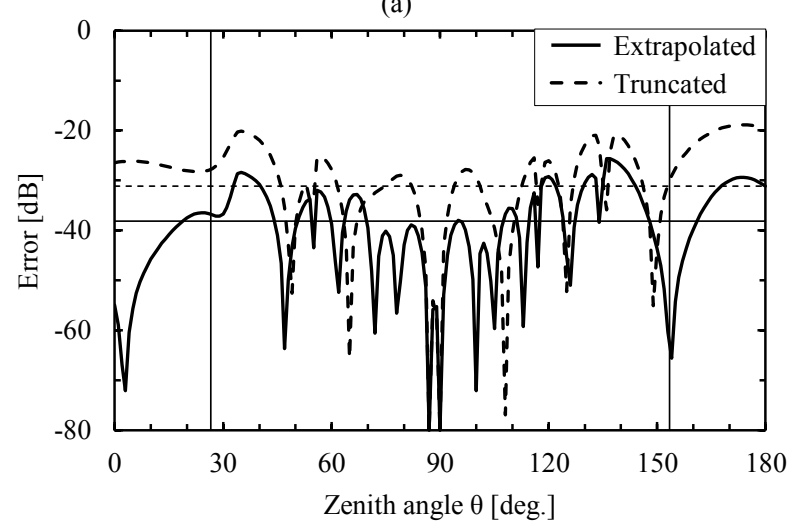

(b)

Fig. 6. Estimated results of $E_{\phi}$ in vertical plane, probe distance $d=\lambda$. (a) is the far-field pattern (solid: reference; chain: truncated; dotted: extrapolated). (b) is the relative error (solid: extrapolated; dotted: truncated). The horizontal lines in (b) present mean value in valid angle region and the region between vertical lines is valid angle region.

extrapolated near-field data, the sidelobe also matches well. The proposed method is able to well represent the reference horizontal plane far-field $\pm 90^{\circ}$ around the main beam. There is a supporting metal pillar behind the AUT; the pattern in the backward direction is disturbed by the reflection wave from there. As the objective of the proposed method is to obtain directivity in maximum direction quickly and accurately, the error in the hind direction with small radiation intensity does not become a problem. Table II shows estimation error of the maximum directivity. The directivity of the AUT can be accurately estimated at any frequency, the maximum error is within $0.2 \mathrm{~dB}$. Relative error of measurement results in the vertical plane are shown in Fig. 11. In the valid angle area, the relative errors of the extrapolated cases are improved against the truncated. The mean values and its improved value are shown in Table III. The improvement by the proposed simple extrapolation method is obvious; at least the average error can be improved by $3 \mathrm{~dB}$ or more. It was shown that the farfield of electrically long antennas can be estimated quickly and accurately by measurement in the minimum space.

\section{CONCLUSION}

A fast method to estimate far-field pattern of electrically long aperture antennas by orthogonal single cut measurement 


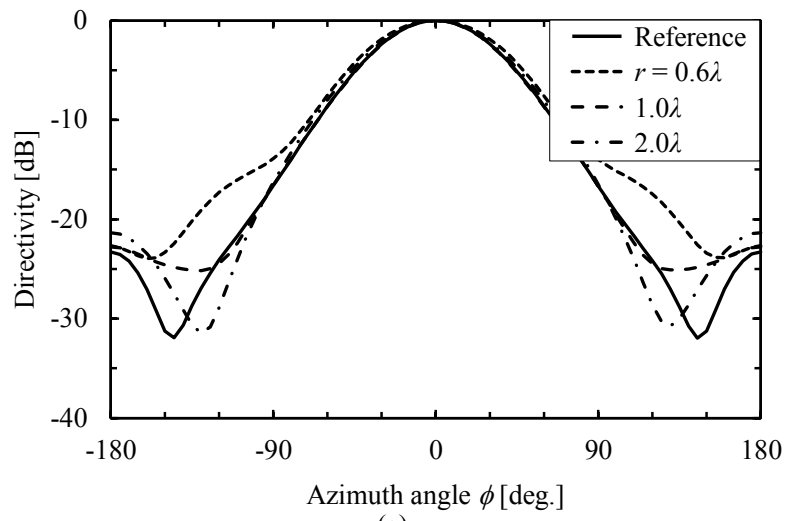

(a)

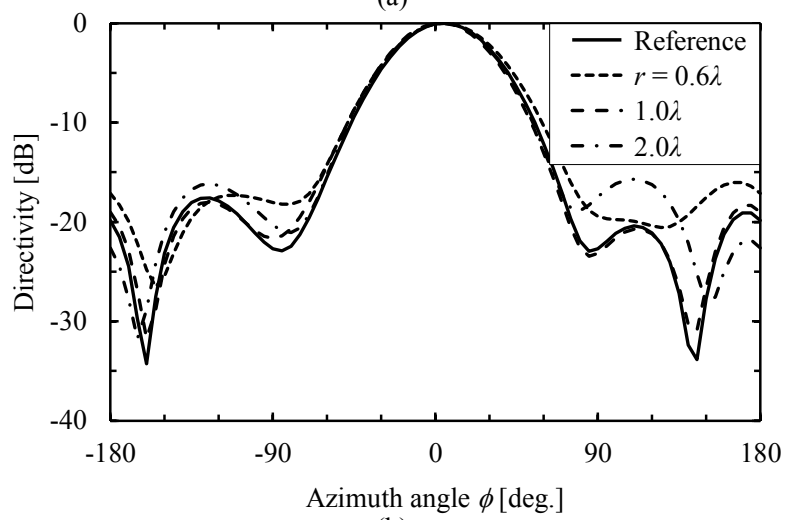

(b)

Fig. 7. Far-field pattern in horizontal plane (solid: reference; dotted: $r=$ $0.6 \lambda$; chain: $r=1.0 \lambda$; break: $r=2.0 \lambda$;). (a) is $E_{\theta}$ of vertical polarized AUT. (b) is $E_{\phi}$ of horizontal polarized AUT.

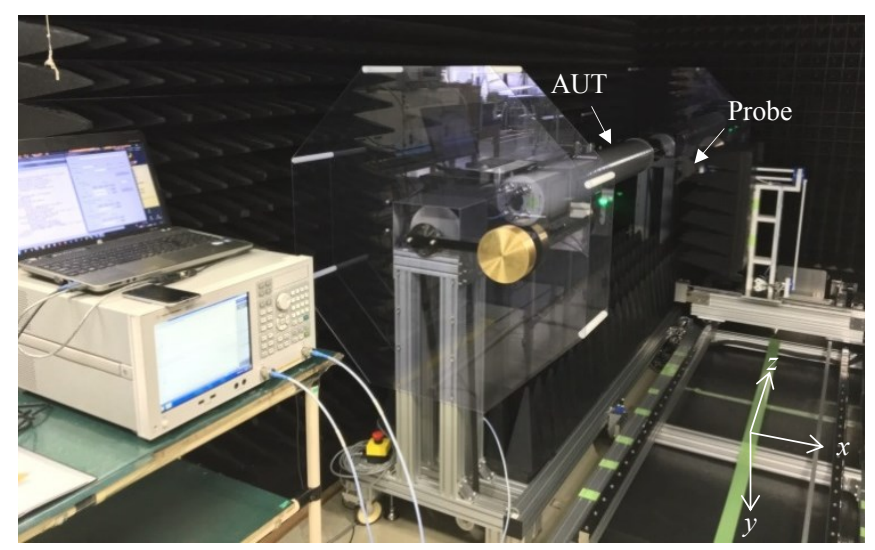

Fig. 8. Measurement overview. The AUT can rotate in $x y$ plane, and the probe can slide in $z$-axis direction.

in compact space was proposed. A method to estimate the far-field pattern of a long aperture array antenna by simple measurement has been proposed. The method is based on the equivalent electric current approach and using orthogonal linear and circular current distribution. In the proposed method, the equivalent current in the vertical plane with insufficient measured length is extrapolated numerically and the estimation accuracy of the far-field in the vertical plane is improved. The proposed method can measure the near-field with a physical

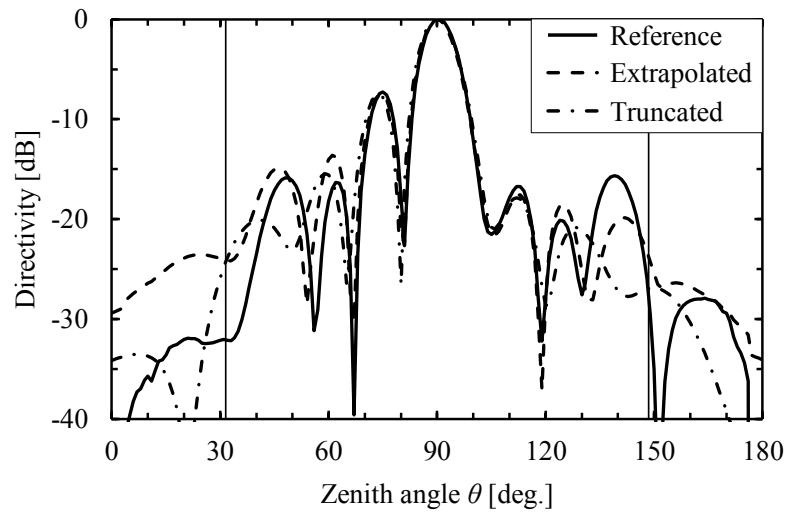

(a)

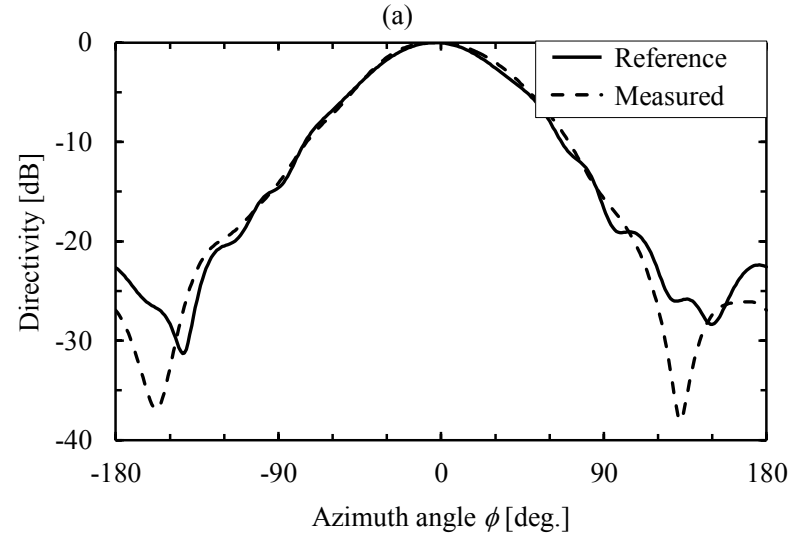

(b)

Fig. 9. Measurement result at $0.79 \mathrm{GHz}$. (a) is vertical plane (solid: reference; chain: truncated; dotted: extrapolated), region between vertical solid lines represents valid angle area after data extension. (b) is horizontal plane (solid: reference; dotted: estimated).

TABLE I

MEASUREMENT SPECIFICATIONS

\begin{tabular}{|c|c|c|c|}
\hline \multirow{7}{*}{$\begin{array}{l}900 \mathrm{MHz} \\
\text { sector } \\
\text { antenna }\end{array}$} & \multirow{3}{*}{$1 \mathrm{D}$ measurement } & Length & $2155 \mathrm{~mm}(6.90 \lambda)$ \\
\hline & & Distance & $100 \mathrm{~mm}(0.32 \lambda)$ \\
\hline & & Interval & $33 \mathrm{~mm}(0.11 \lambda)$ \\
\hline & \multirow{2}{*}{$\begin{array}{l}\text { Circular } \\
\text { measurement }\end{array}$} & Radius & $660 \mathrm{~mm}(2.11 \lambda)$ \\
\hline & & Step & $2^{\circ}(0.07 \lambda)$ \\
\hline & Fresnel region & & $>3500 \mathrm{~mm}$ \\
\hline & Far-field & & $>29700 \mathrm{~mm}$ \\
\hline \multirow{7}{*}{$\begin{array}{l}2.6 \mathrm{GHz} \\
\text { sector } \\
\text { antenna }\end{array}$} & \multirow{3}{*}{ 1D measurement } & Length & $834 \mathrm{~mm}(7.75 \lambda)$ \\
\hline & & Distance & $100 \mathrm{~mm}(0.90 \lambda)$ \\
\hline & & Interval & $11 \mathrm{~mm}(0.10 \lambda)$ \\
\hline & \multirow{2}{*}{$\begin{array}{l}\text { Circular } \\
\text { measurement }\end{array}$} & Radius & $300 \mathrm{~mm}(2.69 \lambda)$ \\
\hline & & Step & $1^{\circ}(0.05 \lambda)$ \\
\hline & Fresnel region & & $>1390 \mathrm{~mm}$ \\
\hline & Far-field & & $>12056 \mathrm{~mm}$ \\
\hline
\end{tabular}

minimum measurement space by using a small loop probe with small electromagnetic disturbance. From the numerical examples, the proposed method demonstrated that it can estimate the far-field using equivalent electrical current data 

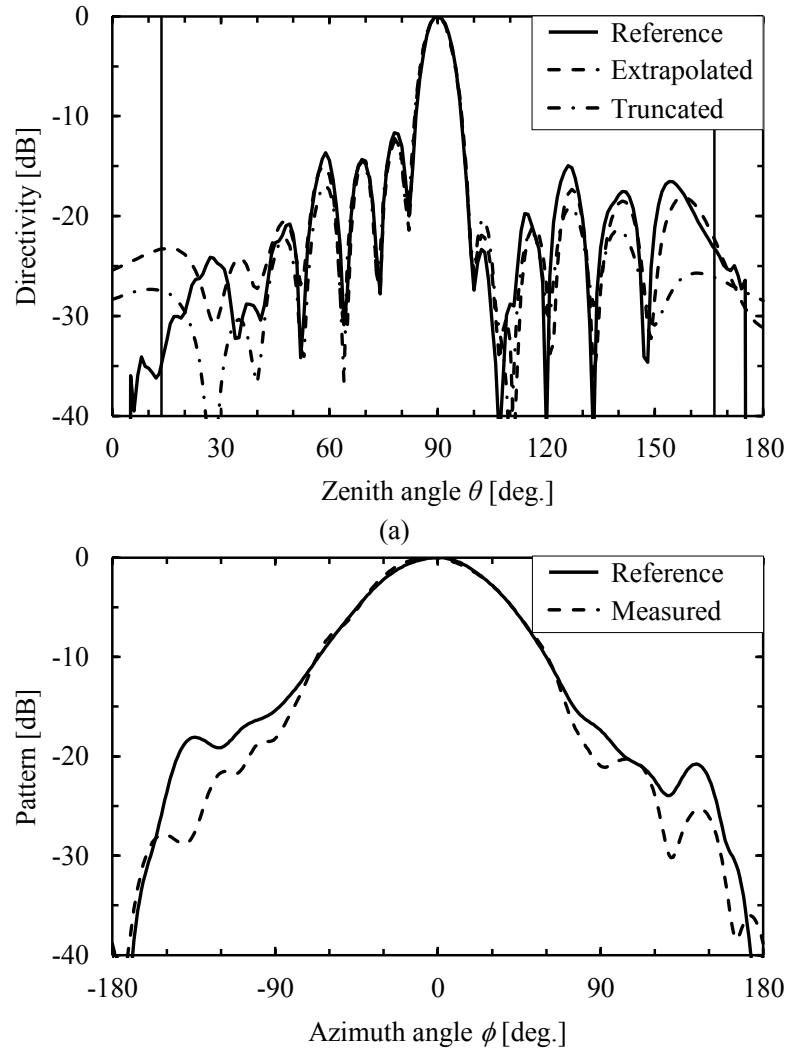

(b)

Fig. 10. Measurement result at $2.69 \mathrm{GHz}$. (a) is vertical plane (solid: reference; chain: truncated; dotted: extrapolated), region between vertical solid lines represents valid angle area after data extension. (b) is horizontal plane (solid: reference; dotted: estimated).

TABLE II

ESTIMATED DIRECTIVITY AND ITS DIFFERENCE

\begin{tabular}{l|rrr}
\hline Freq. [GHz] & Reference [dBi] & Estimated [dBi] & Difference [dB] \\
\hline 0.79 & 16.15 & 16.23 & 0.08 \\
0.81 & 16.47 & 16.59 & 0.12 \\
0.89 & 16.88 & 16.99 & 0.11 \\
0.96 & 17.18 & 17.29 & 0.11 \\
2.49 & 17.51 & 17.64 & 0.13 \\
2.59 & 17.70 & 17.79 & 0.09 \\
2.69 & 18.00 & 18.08 & 0.08 \\
\hline
\end{tabular}

sampled in the very near-field area. We verified the validity of the calculation by using the measured equivalent current distribution. As the maximum directivity can be estimated with an error within $0.2 \mathrm{~dB}$, it is indicated that proposed method is effective for quick and compact far-field estimation of electrically long antennas.

\section{APPENDIX. A}

We prove the estimation equation in the horizontal plane in (9). A circular electric and magnetic equivalent current of radius $\mathrm{r}$ in the $x y$-plane is assumed, and an electro-

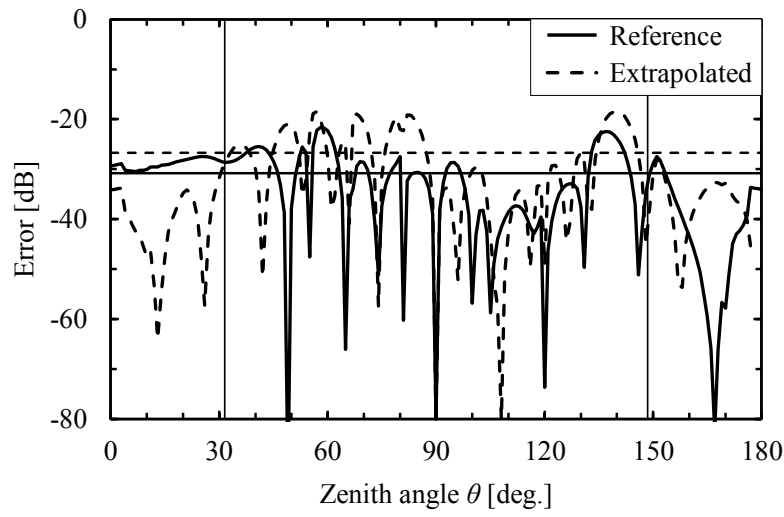

(a)

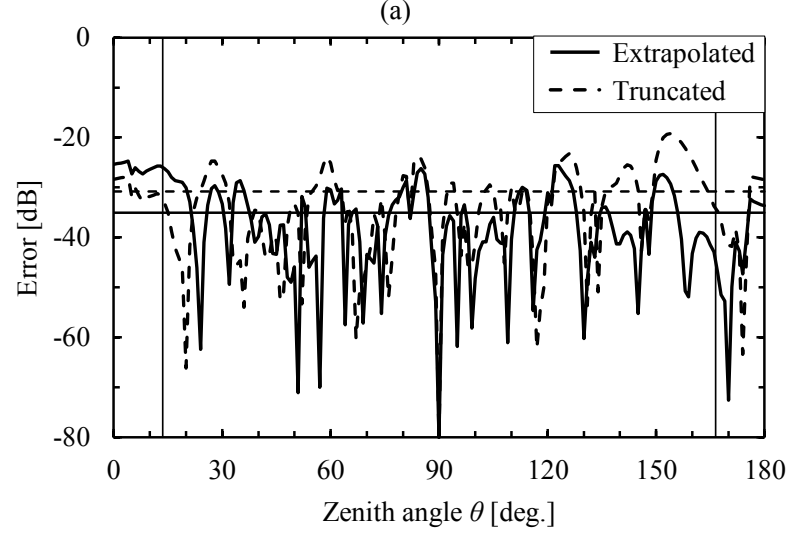

(b)

Fig. 11. Relative error in vertical plane (solid: extrapolated; dotted: truncated), region between vertical solid lines represents valid angle area after data extension, horizontal lines are its mean value. (a) is AUT at $0.79 \mathrm{GHz}$. (b) is AUT at $2.69 \mathrm{GHz}$

TABLE III

MeAn ERror in Vertical Plane

\begin{tabular}{l|rrr}
\hline Freq. [GHz] & Truncated [dB] & Extrapolated [dB] & Improved [dB] \\
\hline 0.79 & -26.75 & -30.83 & 4.08 \\
0.81 & -25.88 & -29.46 & 3.58 \\
0.89 & -26.36 & -30.46 & 4.10 \\
0.96 & -27.31 & -32.94 & 5.63 \\
2.49 & -32.12 & -37.43 & 5.31 \\
2.59 & -29.81 & -35.22 & 5.41 \\
2.69 & -30.84 & -35.07 & 4.23 \\
\hline
\end{tabular}

magnetic equivalent current at point $\phi=0^{\circ}$ (position of $x_{i}=r \cos \phi, y_{i}=r \sin \phi$ ) is focused on. The far-field pattern in horizontal plane of $\theta$ and $\phi$ components by this equivalent EM current are given as

$$
\begin{aligned}
\boldsymbol{E}_{\theta}(\phi) & =\left\{Z_{0} \boldsymbol{J}_{z} e^{\left(j k_{0} d\right)}+\cos \phi \boldsymbol{M}_{\phi} e^{\left(j k_{0} d\right)}\right\} \\
\boldsymbol{E}_{\phi}(\phi) & =\left\{Z_{0} \cos \phi \boldsymbol{J}_{\phi} e^{\left(j k_{0} d\right)}+\boldsymbol{M}_{z} e^{\left(j k_{0} d\right)}\right\} . \\
d & =x_{i} \cos \phi+y_{i} \sin \phi .
\end{aligned}
$$




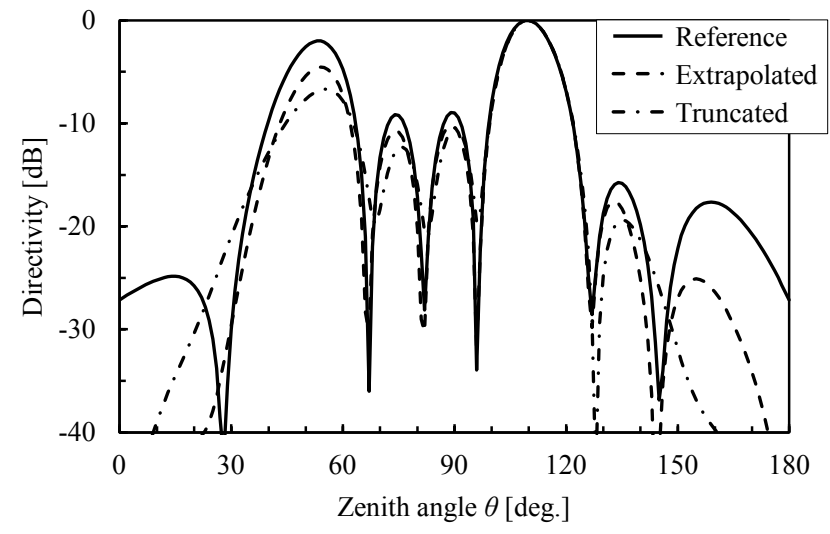

Fig. B.1. Estimated far-field in vertical plane of beam-tilt antenna (solid: reference; chain: truncated; dotted: extrapolated).

Where we can suppose $\boldsymbol{M}=Z_{0} \hat{\mathrm{n}} \times \boldsymbol{J}$ the same as section II, (A.1) gives following equations

$$
\begin{aligned}
\boldsymbol{E}_{\theta}(\phi) & =Z_{0}\left\{\boldsymbol{J}_{z} e^{\left(j k_{0} d\right)}+\cos \phi \boldsymbol{J}_{z} e^{\left(j k_{0} d\right)}\right\} \\
& =Z_{0}(1+\cos \phi) \boldsymbol{J}_{z} e^{\left(j k_{0} d\right)}, \\
\boldsymbol{E}_{\phi}(\phi) & =Z_{0}\left\{\cos \phi \boldsymbol{J}_{\phi} e^{\left(j k_{0} d\right)}+\boldsymbol{J}_{\phi} e^{\left(j k_{0} d\right)}\right\} \\
& =Z_{0}(1+\cos \phi) \boldsymbol{J}_{\phi} e^{\left(j k_{0} d\right)} .
\end{aligned}
$$

By integrating on the horizontal circle, we obtain far-field in the horizontal plane (9).

\section{APPENDIX. B}

We indicate the validity of the proposed method against to beam-tilt antenna. The estimated far-field pattern in vertical plane is shown in Fig. B.1. We use a patch array the same as the Fig. 5, the beam was tilted by phase gradient was given to the feeding points. The proposed method can accurately estimate the tilted main beam and we can see that the developed method is effective for beam-tilt antenna. Due to insufficient measurement length, the estimated level of the grating lobe around $\theta=50^{\circ}$ is degraded. If it can be assumed beforehand that the tilt angle of the AUT is large, it is effective to make the acquisition position of the linear equivalent current in close to the AUT or elongate the measurement length.

\section{REFERENCES}

[1] T. Uno and S. Adachi, "Rante distance requirements for large antenna measurements," IEEE Trans., Antennas and Propag., vol. 37, no. 6, pp.707-720, June 1989.

[2] D. Slater, Near-field antenna measurements, Artech House, 1991.

[3] K. Fujimoto and J. R. James, Mobile Antenna Systems Handbook, Artech House, 1994.

[4] H. Arai and K. Cho, "Cellular and PHS base station antenna system," IEICE Trans., Commun., vol. E86-B, no. 3, pp. 980-992, March 2003.

[5] A. D. Yaghjian, "An overview of near-field antenna measurements," IEEE Trans., Antennas and Propag., vol. AP-34, no. 1, pp. 30-45, Jan. 1986.

[6] R. Conelius, T. Salmerón-Ruiz, F. Saccardi, L. Foged, D. Heberling and M. Sierra-Casteñer, "A comparison of different methods for fast single-cut near-to far-field transformation," IEEE Antennas and Propag. Magazine, vol. 56, no. 2, pp. 252-261, Apr. 2014.
[7] L. J. Forged, G. Barone and F. Saccardi, "Antenna measurement systems using multi-probe technology," 2015 IEEE International Conference on Antenna Measurements \& Applications (2015 CAMA), Dec. 2015.

[8] T. Salmerón-Ruiz, M. Sierra-Casteñer, F. Saccardi, S. Burgos, F. J. CanoFácila and Lars. J. Foged, "A fast single cut spherical near-field-to-farfield transformation using cylindrical modes," European Conference on Antennas and Propagation (EuCap 2014), pp. 2476-2480, Apr. 2014.

[9] J. C. Bolomey, O. M. Bucci, L. Casavola, G. D’Elia, M. D. Migliore and A. Ziyyat, "Reduction of truncation error in near-field measurement of antennas of base-station mobile communication systems," IEEE Trans., Antennas and Propag., vol. 52, no. 2, pp. 593-602, Feb. 2004.

[10] P. Petre and T. K. Sarkar, "Planar near-field to far-field transformation using an equivalent magnetic current approach," IEEE Trans., Antennas and Propag., vol.40, no.11, pp.1348-1356, Nov. 1992.

[11] T. P. Sakar and A. Taaghol, "Near-field to near/far-field transformation for arbitrary near-field geometry utilizing an equivalent electric current and MoM," IEEE Trans., Antennas and Propag., vol. 47, no. 3, pp. 566-573. March 1999.

[12] Y. Álvarez, M. Rodríguez, F. L. Heras and M. M. Hernando, "On the use of the source reconstruction method for estimating radiated EMI in electronic circuits," IEEE Trans., Instrum. Meas., vol. 59, no. 12, pp.3174-3183, Dec. 2010.

[13] W. J. Zhao, H. B. Park, M. Tan, H. H. Park, E. X. Liu, E. Song and E. P. Li, "Far-field prediction from amplitude-only near-field measurements using equivalent electric currents," 2012 IEEE International Symposium on Electromagnetic Compatibility (EMC 2012), pp. 590-593, Aug. 2012.

[14] X. Gao, J. Fan, Y. Zhang, H. Kajbaf and D. Pommerenke, "Far-field prediction using only magnetic near-field scanning for EMI test," IEEE Trans., Antennas and Propag., vol. 56, no. 6, pp. 1335-1343, Dec. 2014.

[15] K. Sarabandi J. Choi, A. Sabet and K. Sabet, "Pattern and gain characterization using nonintrustive very-near-field electro-optical measurements over arbitrary closed surfaces," IEEE Trans., Electromagn. Compat., vol. 65, no. 2, pp. 489-497, Feb. 2017.

[16] E. Ohashi and H. Arai, "Estimation method of directivity on array antennas by using 1D electric current distribution," International Symposium on Antennas and Propagation (ISAP 2014), pp. 291-292, Dec. 2014.

[17] K. T. Wong, Y. Song, C. J. Fulton, S. Khan and W. Y. Tam, "Electrically longdipole in a collocated orthogonal triad - for direction fiinding and polarization estimation," IEEE Trans., Antennas and Propag., vol. 65, no. 11, pp. 6057-6067. Nov. 2017.

[18] J. Litva, T. K. Y. Lo, Digital beamforming in wireless communications, Artech House, 1996.

[19] S. R. Rengarajan and Y. Rahmat-Samii, "The field equivalence principle: Illustration of the establishment of the non-intuitive null fields," IEEE Antenna Propag. Mag., vol. 42, no. 4, pp. 122-128, May 2013.

[20] Y. Cui, R. Li and P. Wang, "A novel broadband planar antenna for 2G/3GLTE base stations," IEEE Trans., Antennas and Propag., vol. 61, no. 5, pp. 2767-2774, May 2013 .

[21] IEEE Std., 1720-2012, "Recommended practice for near-field antenna measurements,".

[22] IEEE Std., 149-1979, "Test procedures for antennas,".

[23] A. C. Newell, C. F. Stubenrauch and R. C. Baird "Calibration of microwave antenna gain standards," Proc. of the IEEE, vol. 74, no. 1, pp. 129-132, Jan. 1986.

[24] FEKO Suite 7.1, http://www.feko.info/

[25] Y. Sugimoto, E. Ohashi and H. Arai, "Far-field pattern estimation on long array antenna by 1D equivalent electric current distribution," 2015 IEEE International Conference on Antenna Measurement \& Applications (CAMA), Dec. 2015.

[26] J. A. Hansen, "On cylindrical near-field scanning techniques," IEEE Trans., Antennas and Propag., vol. AP-28, no. 2, pp. 231-234, March 1980. 


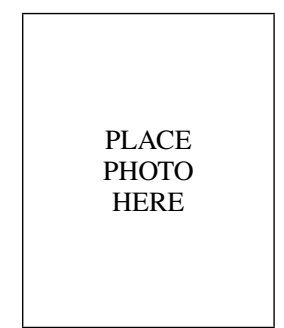

Yoshiki Sugimoto was born in Fukui, Japan. He received his B.S. and M.S. degrees from the University of Fukui, Japan in 2013 and 2015. He is currently working towards a Ph.D. degree in the Division of Physics, Electrical and Computer Engineering, Graduate School of Engineering, Yokohama National University. His research interest includes near-field antenna measurements.

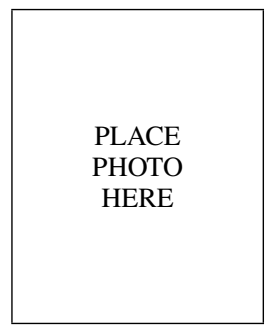

Masanobu Hirose Biography text here.

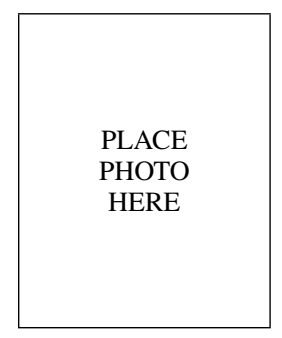

respectively, at Yonsei University, Seoul.

He investigated microwave passive components for high power handling applications such as RF plasma heating in large Tokamaks. He developed a flat diversity antenna for mobile telephone terminal, a polarization diversity base station antenna for Japanese PDC systems, small base station antennas of In-building micro cellular system and DOA estimation for cellular system. His current research interest includes MIMO antennas, wireless power transmission, energy harvesting in EM waves and EMC/EMI antennas.

He received the Young Engineers Award from the IEICE of Japan in 1989 and the "Meritorious Award on Radio" by the Association of Radio Industries and Businesses in 1997 for the development of polarization diversity antenna, in 2006 for the development of DOA estimation system, and in 2011 for the development of light weight phantom.

He was editor-in-chief of IEICE Transactions on Communications, 20052007, and was chair of IEEE AP-S Japan Chapter, 2009-2010. He was associate editor of IEEE Transactions on Antenna and Propagation, 20112013, and chair of Technical Group on Antennas and Propagation of IEICE 2013-2014. He is Fellow of IEEE and IEICE.

$\mathrm{He}$ is the author of five edited books, three research book chapters, over 140 journal papers and 1100 conference papers.

Toshiyuki Maruyama Biography text here.

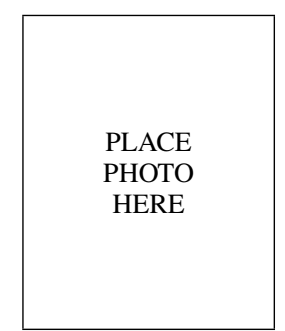

Satoru Kurokawa Biography text here.
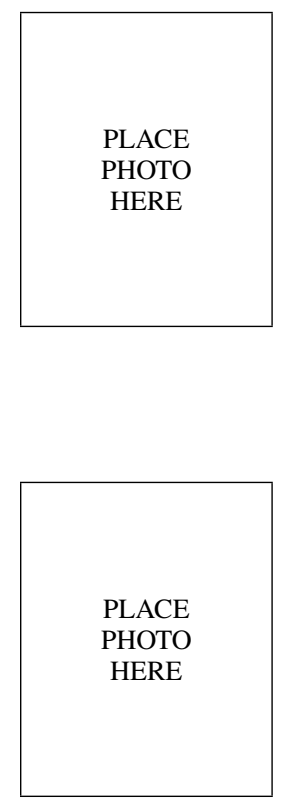

Masahiko Nasuno Biography text here. 